\title{
NOTES ON THE SUBSPACE PERTURBATION PROBLEM FOR OFF-DIAGONAL PERTURBATIONS
}

\author{
ALBRECHT SEELMANN \\ (Communicated by Michael Hitrik)
}

\begin{abstract}
The variation of spectral subspaces for linear self-adjoint operators under an additive bounded off-diagonal perturbation is studied. To this end, the optimization approach for general perturbations in [J. Anal. Math., to appear; arXiv:1310.4360 (2013)] is adapted. It is shown that, in contrast to the case of general perturbations, the corresponding optimization problem cannot be reduced to a finite-dimensional problem. A suitable choice of the involved parameters provides an upper bound for the solution of the optimization problem. In particular, this yields a rotation bound on the subspaces that is stronger than the previously known one from [J. Reine Angew. Math. 708 (2015), 1-15].
\end{abstract}

\section{INTRODUCTION}

The present work deals with a particular case of the subspace perturbation problem previously discussed in several recent works such as [2, 6, 9].

For the whole note let $A$ be a self-adjoint possibly unbounded operator on a separable Hilbert space $\mathcal{H}$ such that the spectrum of $A$ is separated as

$$
\operatorname{spec}(A)=\sigma \cup \Sigma \quad \text { with } \quad d:=\operatorname{dist}(\sigma, \Sigma)>0 .
$$

Moreover, let $V$ be a bounded self-adjoint operator on $\mathcal{H}$, and suppose that $V$ is off-diagonal with respect to the decomposition $\mathcal{H}=\operatorname{Ran} \mathrm{E}_{A}(\sigma) \oplus \operatorname{Ran} \mathrm{E}_{A}(\Sigma)$, that is,

$$
\mathrm{E}_{A}(\sigma) V \mathrm{E}_{A}(\sigma)=0=\mathrm{E}_{A}(\Sigma) V \mathrm{E}_{A}(\Sigma) .
$$

Here, $\mathrm{E}_{A}$ denotes the spectral measure for the self-adjoint operator $A$.

In this situation, it has been shown in [12, Proposition 2.5.22] (see also [7, Theorem 1.3] for the case of bounded operators $A$ ) that

$$
\operatorname{spec}(A+V) \subset \overline{\mathcal{O}_{\delta_{V}}(\operatorname{spec}(A))} \quad \text { with } \quad \delta_{V}:=\|V\| \tan \left(\frac{1}{2} \arctan 2 \frac{\|V\|}{d}\right),
$$

where $\mathcal{O}_{\delta_{V}}(\operatorname{spec}(A))$ denotes the open $\delta_{V}$-neighbourhood of the spectrum of $A$. In particular, if

$$
\|V\|<\frac{\sqrt{3}}{2} d
$$

Received by the editors October 26, 2015.

2010 Mathematics Subject Classification. Primary 47A55; Secondary 47A15, 47B15.

Key words and phrases. Subspace perturbation problem, spectral subspaces, maximal angle between closed subspaces, off-diagonal perturbations. 
that is, $\delta_{V}<d / 2$, then the spectrum of $A+V$ is likewise separated into two disjoint components, each contained in the open $d / 2$-neighbourhood of $\sigma$ and $\Sigma$, respectively. This gap non-closing condition on $\|V\|$ is known to be sharp; see [7. Example 1.5].

The variation of the corresponding spectral subspaces under the perturbation can be measured by the associated maximal angle

$$
\theta:=\arcsin \left(\left\|\mathrm{E}_{A}(\sigma)-\mathrm{E}_{A+V}\left(\mathcal{O}_{d / 2}(\sigma)\right)\right\|\right) .
$$

Here, it is a natural question whether the condition (1.3) is sufficient to ensure that $\theta<\pi / 2$. More specifically, one is interested in the best possible constant $c_{\text {opt-off }} \in\left(0, \frac{\sqrt{3}}{2}\right]$ such that

$$
\theta<\frac{\pi}{2} \quad \text { whenever } \quad\|V\|<c_{\text {opt-off }} \cdot d
$$

It is worth mentioning that the situation looks slightly different if the two sets $\sigma$ and $\Sigma$ are additionally assumed to be subordinated, that is, $\sup \sigma<\inf \Sigma$ or vice versa, or if one of the two sets lies in a finite gap of the other one. In these particular cases, the gap non-closing condition on $\|V\|$ can be relaxed considerably, and, at the same time, these relaxed conditions are also known to ensure that the associated maximal angle $\theta$ is strictly less than $\pi / 2$. A short survey of the corresponding results can be found, e.g., in [3].

Under the assumption (1.1) alone, however, the value of $c_{\text {opt-off }}$ in (1.4) is unknown. It has been conjectured to be $\sqrt{3} / 2=0.8660254 \ldots$ (see [7]), the same constant as in the gap non-closing condition, but no proof for this is available yet.

The analogous problem for general, not necessarily off-diagonal, perturbations has been discussed in $2,6,6,8,9$. In this more general setting, the (sharp) gap nonclosing condition is known to be $\|V\|<d / 2$, and it is likewise an open problem whether the best possible constant $c_{\mathrm{opt}} \in\left(0, \frac{1}{2}\right]$ corresponding to (1.4) satisfies $c_{\mathrm{opt}}=1 / 2$. The currently best known lower bound $c_{\mathrm{opt}} \geq c_{\text {crit }}$ with an explicit constant $c_{\text {crit }}=0.4548 \ldots$ was obtained in the author's work 9 . The present note ties in with the considerations there.

As a direct consequence of the results for general perturbations, one clearly has $c_{\text {opt-off }} \geq c_{\text {opt }}$. In particular, the maximal angle $\theta$ satisfies the bound

$$
\theta \leq \frac{1}{2} \arcsin \left(\pi \frac{\|V\|}{d}\right) \quad \text { for } \quad\|V\| \leq \frac{d}{\pi},
$$

proved in [10, Corollary 2] (see also [2, Corollary 4.3 and Remark 4.4]). For offdiagonal perturbations $V$, this bound agrees with the one obtained by combining [1. Theorems 3.6 (i) and 7.6] and [5, Corollary 3.4].

Making use of the off-diagonal structure of the perturbation, it was shown in [8. Theorem 3.3] that

$$
\theta \leq \frac{\pi}{2} \int_{0}^{\frac{\|V\|}{d}} \frac{\mathrm{d} \tau}{1-2 \tau \tan \left(\frac{1}{2} \arctan (2 \tau)\right)}<\frac{\pi}{2} \quad \text { for } \quad\|V\|<c_{\text {off }} \cdot d,
$$

where $c_{\text {off }}=0.6759893 \ldots$ is determined by

$$
\int_{0}^{c_{\text {off }}} \frac{\mathrm{d} \tau}{1-2 \tau \tan \left(\frac{1}{2} \arctan (2 \tau)\right)}=1
$$

In particular, this yields the stronger lower bound $c_{\text {opt-off }} \geq c_{\text {off. }}$. An earlier, slightly weaker result can be found in [7, Theorem 2.2]. 
It should be noted that the result (1.6) was originally formulated only for the case where the operator $A$ is assumed to be bounded, but it can easily be extended to the unbounded case. For the sake of completeness, a corresponding proof is reproduced in Remark 2.1 below.

In their recent survey article [3], Albeverio and Motovilov have stated that $c_{\text {opt-off }}>0.692834$, based on the iteration approach from [2] and [9] adapted to the case of off-diagonal perturbations.

In the present note this approach is refined. The principal result is that

$$
c_{\text {opt-off }}>0.6940725 \text {, }
$$

which, together with a corresponding bound on the maximal angle, is obtained by a suitable choice of the involved parameters; see Theorem 2.5 below. We also show that, in contrast to the case of general perturbations in [9], the optimization problem for these parameters cannot be reduced to a finite-dimensional problem; see Proposition 2.3 below. In fact, this optimization problem is not solved explicitly yet. Nevertheless, the result presented here is the strongest one obtained for this problem so far.

\section{The optimization PROBLEM FOR OFF-DiAgONAL PERTURBATIONS}

Suppose that the off-diagonal perturbation $V$ is non-trivial, that is, $V \neq 0$. For $0 \leq t<\sqrt{3} / 2$, introduce $B_{t}:=A+t d \cdot V /\|V\|$ on $\operatorname{Dom}\left(B_{t}\right):=\operatorname{Dom}(A)$, and denote by $P_{t}:=\mathrm{E}_{B_{t}}\left(\mathcal{O}_{d / 2}(\sigma)\right)$ the spectral projection for $B_{t}$ associated with the open $d / 2$-neighbourhood $\mathcal{O}_{d / 2}(\sigma)$ of $\sigma$.

Clearly, one has $\left\|B_{t}-A\right\|=t d<\sqrt{3} d / 2$. As described in Section 1, this implies that the spectrum of the perturbed operator $B_{t}$ is separated as

$$
\operatorname{spec}\left(B_{t}\right)=\omega_{t} \cup \Omega_{t}
$$

with

$$
\omega_{t}=\operatorname{spec}\left(B_{t}\right) \cap \overline{\mathcal{O}_{\delta_{t} \cdot d}(\sigma)} \quad \text { and } \quad \Omega_{t}=\operatorname{spec}\left(B_{t}\right) \cap \overline{\mathcal{O}_{\delta_{t} \cdot d}(\Sigma)} \text {, }
$$

where

$$
\delta_{t}:=t \tan \left(\frac{1}{2} \arctan 2 t\right)=\frac{1}{2} \sqrt{1+4 t^{2}}-\frac{1}{2}<\frac{1}{2} .
$$

In particular, for $0 \leq t<\sqrt{3} / 2$ one has $P_{t}=\mathrm{E}_{B_{t}}\left(\omega_{t}\right)$ and

$$
\operatorname{dist}\left(\omega_{t}, \Omega_{t}\right) \geq\left(1-2 \delta_{t}\right) d=\left(2-\sqrt{1+4 t^{2}}\right) d .
$$

Let $t \in\left(0, \frac{\sqrt{3}}{2}\right)$, and let $0=t_{0}<\cdots<t_{n+1}=t$ with $n \in \mathbb{N}_{0}$ be a finite partition of the interval $[0, t]$. As in 2 and 9 , the triangle inequality for the maximal angle (see, e.g., 4, Corollary 4]) yields

$$
\arcsin \left(\left\|P_{0}-P_{t}\right\|\right) \leq \sum_{j=0}^{n} \arcsin \left(\left\|P_{t_{j}}-P_{t_{j+1}}\right\|\right) .
$$

Moreover, considering $B_{t_{j+1}}=B_{t_{j}}+\left(t_{j+1}-t_{j}\right) d \cdot V /\|V\|$ as a perturbation of $B_{t_{j}}$ and taking into account the a priori bound (2.1), we observe that

$$
\frac{\left\|B_{t_{j+1}}-B_{t_{j}}\right\|}{\operatorname{dist}\left(\omega_{t_{j}}, \Omega_{t_{j}}\right)} \leq \frac{t_{j+1}-t_{j}}{1-2 \delta_{t_{j}}}=: \lambda_{j} \quad \text { for } \quad j=0, \ldots, n \text {. }
$$


In particular, the bound (1.5) for general perturbations implies that

$$
\arcsin \left(\left\|P_{t_{j}}-P_{t_{j+1}}\right\|\right) \leq \frac{1}{2} \arcsin \left(\pi \lambda_{j}\right) \quad \text { whenever } \quad \lambda_{j} \leq \frac{1}{\pi} .
$$

For partitions of the interval $[0, t]$ with arbitrarily small mesh size, this allows one to reproduce the bound (1.6) from 8 :

Remark 2.1 (cf. [9, Section 2]). If the mesh size of the partition of the interval $[0, t]$ is sufficiently small, then the Riemann sum

$$
\sum_{j=0}^{n} \lambda_{j}=\sum_{j=0}^{n} \frac{t_{j+1}-t_{j}}{1-2 \delta_{t_{j}}}
$$

is close to the integral $\int_{0}^{t} \frac{\mathrm{d} \tau}{1-2 \delta_{\tau}}$. Since, at the same time, each $\lambda_{j}$ is small and $\arcsin (x) / x \rightarrow 1$ as $x \rightarrow 0$, we conclude from (2.2) and (2.4) that

$$
\arcsin \left(\left\|P_{0}-P_{t}\right\|\right) \leq \frac{\pi}{2} \int_{0}^{t} \frac{\mathrm{d} \tau}{1-2 \delta_{\tau}} .
$$

Taking into account that $A+V=B_{t}$ for $t=\|V\| / d$, this agrees with (1.6).

Clearly, the same reasoning for the interval $[s, t]$ can be used to show that

$$
\arcsin \left(\left\|P_{s}-P_{t}\right\|\right) \leq \frac{\pi}{2} \int_{s}^{t} \frac{\mathrm{d} \tau}{1-2 \delta_{\tau}} \quad \text { for } \quad 0 \leq s<t<\frac{\sqrt{3}}{2} .
$$

It turns out that the bound (1.6) on the maximal angle is stronger than (1.5); see Lemma 2.2 (a) below. However, part (b) of the same lemma indicates that the situation changes when the estimate on the maximal angle is iterated.

\section{Lemma 2.2.}

(a) One has

$$
\frac{\pi}{2} \int_{0}^{s} \frac{\mathrm{d} \tau}{1-2 \delta_{\tau}}<\frac{1}{2} \arcsin (\pi s) \quad \text { for } \quad 0<s \leq \frac{1}{\pi} .
$$

(b) For every $0<r<\sqrt{3} / 2$ there is $\varepsilon>0$ with $\varepsilon \leq\left(1-2 \delta_{r}\right) / \pi$ and $r+\varepsilon<\sqrt{3} / 2$ such that

$$
\frac{1}{2} \arcsin \left(\pi \frac{s-r}{1-2 \delta_{r}}\right)<\frac{\pi}{2} \int_{r}^{s} \frac{\mathrm{d} \tau}{1-2 \delta_{\tau}} \quad \text { for } \quad r<s \leq r+\varepsilon .
$$

Moreover, the number $\varepsilon$ can be chosen independently of $r$ from a compact subinterval of $\left(0, \frac{\sqrt{3}}{2}\right)$.

Proof. Let $r$ with $0 \leq r<\sqrt{3} / 2$ be arbitrary, and define

$$
h_{r}(s):=\frac{\pi}{2} \int_{r}^{s} \frac{\mathrm{d} \tau}{1-2 \delta_{\tau}}-\frac{1}{2} \arcsin \left(\pi \frac{s-r}{1-2 \delta_{r}}\right) .
$$

Taking into account that $1-2 \delta_{\tau}=2-\sqrt{1+4 \tau^{2}}$, one computes

$$
h_{r}^{\prime}(s)=\frac{\pi}{2}\left(\frac{1}{2-\sqrt{1+4 s^{2}}}-\frac{1}{\sqrt{\left(2-\sqrt{1+4 r^{2}}\right)^{2}-\pi^{2}(s-r)^{2}}}\right) .
$$

For $r=0$, the inequality $h_{0}^{\prime}(s)<0$ is equivalent to $\sqrt{1-\pi^{2} s^{2}}<2-\sqrt{1+4 s^{2}}$, and it is easy to verify that the latter is valid for $0<s \leq 1 / \pi$. Since $h_{0}(0)=0$, this implies that $h_{0}(s)<0$ for $0<s \leq 1 / \pi$, which proves (a). 
Now, let $r>0$. In this case, the inequality $h_{r}^{\prime}(s)>0$ is equivalent to

$$
\left(2-\sqrt{1+4 r^{2}}\right)^{2}-\pi^{2}(s-r)^{2}>\left(2-\sqrt{1+4 s^{2}}\right)^{2},
$$

which, in turn, can be rewritten as

$$
4\left(s^{2}-r^{2}\right)\left(\frac{4}{\sqrt{1+4 r^{2}}+\sqrt{1+4 s^{2}}}-1\right)>\pi^{2}(s-r)^{2} .
$$

Dividing the latter inequality for $s>r$ by $s-r$ and then letting $s$ approach $r$, one arrives at the inequality

$$
8 r \cdot\left(\frac{2}{\sqrt{1+4 r^{2}}}-1\right)>0
$$

which is obviously valid for $0<r<\sqrt{3} / 2$. Hence, by continuity, one concludes that $h_{r}^{\prime}(s)>0$ if $s>r$ is sufficiently close to $r$. Since $h_{r}(r)=0$, this proves the first claim in (b). The second claim that $\varepsilon$ can be chosen independently of $r$ from a compact subinterval of $\left(0, \frac{\sqrt{3}}{2}\right)$ follows by the same reasoning and the fact that the left-hand side of (2.6) is bounded away from 0 for $r$ from a compact subinterval of $\left(0, \frac{\sqrt{3}}{2}\right)$.

The preceding lemma demonstrates one of the main differences between the current case of off-diagonal perturbations and the one of general perturbations. Namely, the function $\tau \mapsto 1-2 \delta_{\tau}$ from the lower bound (2.1) is not affine, which corresponds to the fact that for $B_{s}=B_{r}+\left(B_{s}-B_{r}\right)$ with $0<r<s$ the perturbation $B_{s}-B_{r}$ does not need to be (and usually is not) off-diagonal with respect to the decomposition $\mathcal{H}=\operatorname{Ran} P_{r} \oplus \operatorname{Ran} P_{r}^{\perp}$. By contrast, the corresponding function $\tau \mapsto 1-2 \tau$ for general perturbations is affine. In particular, for $0 \leq r<s<1 / 2$ with $\lambda:=\frac{s-r}{1-2 r} \leq \frac{1}{\pi}$ one has

$$
\frac{\pi}{2} \int_{r}^{s} \frac{\mathrm{d} \tau}{1-2 \tau}=\frac{\pi}{2} \int_{0}^{\lambda} \frac{\mathrm{d} \tau}{1-2 \tau}>\frac{1}{2} \arcsin (\pi \lambda),
$$

cf. [2, Remark 5.5], regardless of whether $r>0$ or $r=0$. The effect expressed by Lemma 2.2 is therefore not present in the case of general perturbations.

In view of (2.2) - (2.5), Lemma 2.2 suggests to estimate $\arcsin \left(\left\|P_{0}-P_{t}\right\|\right)$ as

$$
\arcsin \left(\left\|P_{0}-P_{t}\right\|\right) \leq \frac{\pi}{2} \int_{0}^{\lambda_{0}} \frac{\mathrm{d} \tau}{1-2 \delta_{\tau}}+\frac{1}{2} \sum_{j=1}^{n} \arcsin \left(\pi \lambda_{j}\right),
$$

provided that $\lambda_{j} \leq 1 / \pi$ for $j=1, \ldots, n$ and that $\lambda_{0}=t_{1} \leq c_{\text {off }}$ with $c_{\text {off }}$ as in (1.6). The optimization problem then consists in minimizing the right-hand side of (2.7) for fixed $t \in\left(0, \frac{\sqrt{3}}{2}\right)$ over all corresponding choices of partitions of the interval $[0, t]$. This is the natural adaption of the approach in [2] and [9] to the current situation of off-diagonal perturbations $V$.

The following result shows that this optimization problem, unlike the one in [9], cannot be reduced to a finite-dimensional problem. It is a direct application of Lemma 2.2(b).

Proposition 2.3. For fixed $t \in\left(0, \frac{\sqrt{3}}{2}\right)$, there is no finite partition of the interval $[0, t]$ which minimizes the right-hand side of (2.7). 
Proof. Let $0=t_{0}<\cdots<t_{n+1}=t$ with $n \in \mathbb{N}_{0}$ be an arbitrary partition of the interval $[0, t]$. For every $r \in\left(0, t_{1}\right)$ one has

$$
\int_{0}^{t_{1}} \frac{\mathrm{d} \tau}{1-2 \delta_{\tau}}=\int_{0}^{r} \frac{\mathrm{d} \tau}{1-2 \delta_{\tau}}+\int_{r}^{t_{1}} \frac{\mathrm{d} \tau}{1-2 \delta_{\tau}} .
$$

Since the number $\varepsilon$ in Lemma 2.2(b) can be chosen independently of $r$ from a compact subinterval of $\left(0, \frac{\sqrt{3}}{2}\right)$, we may choose $r \in\left(0, t_{1}\right)$ in such a way that $\frac{t_{1}-r}{1-2 \delta_{r}} \leq \frac{1}{\pi}$ and

$$
\frac{1}{2} \arcsin \left(\pi \frac{t_{1}-r}{1-2 \delta_{r}}\right)<\frac{\pi}{2} \int_{r}^{t_{1}} \frac{\mathrm{d} \tau}{1-2 \delta_{\tau}} .
$$

The refined partition $0=t_{0}<r<t_{1}<\cdots<t_{n+1}=t$ then leads to a righthand side in (2.7) which is strictly less than the one corresponding to the original partition of the interval $[0, t]$.

Remark 2.4. Iterating the argument in the proof of Proposition 2.3 shows that, from the point of view of minimizing the right-hand side of (2.7), one may always assume that $\lambda_{0}=0$. In other words, the first summand of the right-hand side of (2.7) can be replaced by $\frac{1}{2} \arcsin \left(\pi \lambda_{0}\right)$, provided that $\lambda_{0} \leq 1 / \pi$, without affecting the optimization result. In fact, the latter has been considered in $[3$. However, when considering specific finite partitions of the interval $[0, t]$, Lemma[2.2(a) shows that the current approach (2.7) is more suitable.

Another difficulty in the problem of minimizing the right-hand side of (2.7) arises by the fact that, given a partition of the interval $[0, t]$, an efficient explicit representation of $t$ in terms of the corresponding parameters $\lambda_{j}$ is not at hand. In contrast to the case of general perturbations (cf. [9, Section 3]), it is thus unclear how to determine the critical points for the reduced finite-dimensional optimization problems associated with a fixed number of supporting points in the partitions.

In fact, the problem of minimizing the right-hand side of (2.7) is not solved explicitly yet. So far, the author can only guess at a choice of the parameters $\lambda_{j}$ guaranteeing that $c_{\text {opt-off }}>0.694$. In view of Proposition 2.3. this guess seems to be a reasonable compromise between the complexity of the choice of the parameters and the strength of the result:

Let $n=4$. Choose $\lambda_{0} \in\left(0, c_{\text {off }}\right)$ such that

$$
\frac{\pi}{2} \int_{0}^{\lambda_{0}} \frac{\mathrm{d} \tau}{1-2 \delta_{\tau}}=\frac{1}{3}
$$

and $\lambda_{j} \in\left(0, \frac{1}{\pi}\right], j=1, \ldots, 4$, such that $2 \arcsin \left(\pi \lambda_{j}\right)=\frac{\pi}{2}-\frac{1}{3}$, that is,

$$
\lambda_{j}=\frac{1}{\pi} \sin \left(\frac{3 \pi-2}{12}\right)=0.1846204 \ldots \quad \text { for } \quad j=1, \ldots, 4 .
$$

For this choice of $n$ and $\lambda_{j}$ the right-hand side of (2.7) equals $\pi / 2$.

A numerical calculation gives

$$
\tau_{1}:=\lambda_{0}>0.2062031 .
$$

Upon observing that the mapping $\left[0, \frac{\sqrt{3}}{2}\right] \ni \tau \mapsto \tau+\lambda_{1}\left(1-2 \delta_{\tau}\right)$ is strictly increasing, it is then easy to verify that

$$
\tau_{2}:=\tau_{1}+\lambda_{1}\left(1-2 \delta_{\tau_{1}}\right)>0.3757396 .
$$


In the same way, one has

$$
\tau_{3}:=\tau_{2}+\lambda_{2}\left(1-2 \delta_{\tau_{2}}\right)>0.5140409, \tau_{4}:=\tau_{3}+\lambda_{3}\left(1-2 \delta_{\tau_{3}}\right)>0.6184976,
$$

and

$$
c_{\text {off }}^{*}:=\tau_{5}:=\tau_{4}+\lambda_{4}\left(1-2 \delta_{\tau_{4}}\right)>0.6940725 .
$$

Finally, consider the piecewise defined function $N_{\mathrm{off}}^{*}:\left[0, c_{\mathrm{off}}^{*}\right] \rightarrow\left[0, \frac{\pi}{2}\right]$ with

$$
N_{\mathrm{off}}^{*}(t):= \begin{cases}\frac{\pi}{2} \int_{0}^{t} \frac{\mathrm{d} \tau}{1-2 \delta_{\tau}}, & 0 \leq t \leq \tau_{1}, \\ \frac{1}{3}+(j-1) \frac{3 \pi-2}{24}+\frac{1}{2} \arcsin \left(\pi \frac{t-\tau_{j}}{1-2 \delta_{\tau_{j}}}\right), & \tau_{j}<t \leq \tau_{j+1} .\end{cases}
$$

Clearly, the function $N_{\text {off }}^{*}$ is strictly increasing with $N_{\text {off }}^{*}\left(c_{\text {off }}^{*}\right)=\pi / 2$, continuous on $\left[0, c_{\text {off }}^{*}\right]$, and continuously differentiable on $\left(0, c_{\text {off }}^{*}\right) \backslash\left\{\tau_{2}, \tau_{3}, \tau_{4}\right\}$.

We now use $\tau_{1}, \ldots, \tau_{4}$ as supporting points for the partitions of the interval $[0, t]$. More precisely, using the partition $0<\cdots<\tau_{j}<t$ for $\tau_{j}<t \leq \tau_{j+1}$ and the trivial one $0<t$ for $t \leq \tau_{1}$, it follows from (2.7) and (2.9) that

$$
\arcsin \left(\left\|P_{0}-P_{t}\right\|\right) \leq N_{\text {off }}^{*}(t)<\frac{\pi}{2} \quad \text { for } \quad 0 \leq t<c_{\text {off }}^{*} .
$$

Taking into account that $B_{t}=A+V$ with $t=\|V\| / d$, the preceding considerations now summarize to the following theorem, the main result in this note.

Theorem 2.5. Let $A$ be a self-adjoint operator on a separable Hilbert space $\mathcal{H}$ with spectrum separated as in (1.1), and let $V$ be a bounded self-adjoint operator on $\mathcal{H}$ which is off-diagonal with respect to the decomposition $\mathcal{H}=\mathrm{E}_{A}(\sigma) \oplus \mathrm{E}_{A}(\Sigma)$, that is,

$$
\mathrm{E}_{A}(\sigma) V \mathrm{E}_{A}(\sigma)=0=\mathrm{E}_{A}(\Sigma) V \mathrm{E}_{A}(\Sigma) .
$$

If $V$ satisfies

with $c_{\mathrm{off}}^{*}$ as in (2.8), then

$$
\|V\|<c_{\mathrm{off}}^{*} \cdot d
$$

$$
\arcsin \left(\left\|\mathrm{E}_{A}(\sigma)-\mathrm{E}_{A+V}\left(\mathcal{O}_{d / 2}(\sigma)\right)\right\|\right) \leq N_{\text {off }}^{*}\left(\frac{\|V\|}{d}\right)<\frac{\pi}{2},
$$

where the function $N_{\mathrm{off}}^{*}$ is given by (2.9).

It is a direct consequence of Theorem 2.5 that the best possible constant $c_{\text {opt-off }}$ in (1.4) satisfies the lower bound

$$
c_{\text {opt-off }}>c_{\text {off }}^{*}>0.6940725,
$$

where the fact that the first inequality in (2.10) is strict is due to Proposition 2.3. Furthermore, numerical evaluations suggest that the corresponding bound on the maximal angle between the subspaces $\operatorname{Ran} \mathrm{E}_{A}(\sigma)$ and $\operatorname{Ran} \mathrm{E}_{A+V}\left(\mathcal{O}_{d / 2}(\sigma)\right)$ is indeed stronger than the one given by (1.6), that is,

$$
N_{\text {off }}^{*}(t)<\frac{\pi}{2} \int_{0}^{t} \frac{\mathrm{d} \tau}{1-2 \delta_{\tau}} \quad \text { for } \quad \tau_{1}<t \leq c_{\text {off }} .
$$

\section{ACKNOWLEDGEMENTS}

The material presented in this work is part of the author's Ph.D. thesis [11]. The author is grateful to his Ph.D. advisor, Vadim Kostrykin, for fruitful discussions. He would also like to thank Christoph Uebersohn for helpful remarks on the manuscript. 


\section{REFERENCES}

[1] Sergio Albeverio, Konstantin A. Makarov, and Alexander K. Motovilov, Graph subspaces and the spectral shift function, Canad. J. Math. 55 (2003), no. 3, 449-503, DOI 10.4153/CJM2003-020-7. MR.1980611 (2004d:47031)

[2] Sergio Albeverio and Alexander K. Motovilov, Sharpening the norm bound in the subspace perturbation theory, Complex Anal. Oper. Theory 7 (2013), no. 4, 1389-1416, DOI 10.1007/s11785-012-0245-7. MR3079864

[3] S. Albeverio and A. K. Motovilov, Bounds on variation of the spectrum and spectral subspaces of a few-body Hamiltonian, e-print arXiv:1410.3231v1 [math-ph] (2014).

[4] Lawrence G. Brown, The rectifiable metric on the set of closed subspaces of Hilbert space, Trans. Amer. Math. Soc. 337 (1993), no. 1, 279-289, DOI 10.2307/2154322. MR.1155349 (93g:46021)

[5] Vadim Kostrykin, Konstantin A. Makarov, and Alexander K. Motovilov, Existence and uniqueness of solutions to the operator Riccati equation. A geometric approach, Advances in differential equations and mathematical physics (Birmingham, AL, 2002), Contemp. Math., vol. 327, Amer. Math. Soc., Providence, RI, 2003, pp. 181-198, DOI 10.1090/conm/327/05814. MR1991541(2004f:47012)

[6] Vadim Kostrykin, Konstantin A. Makarov, and Alexander K. Motovilov, On a subspace perturbation problem, Proc. Amer. Math. Soc. 131 (2003), no. 11, 3469-3476, DOI 10.1090/S0002-9939-03-06917-X. MR.1991758(2004c:47029)

[7] Vadim Kostrykin, K. A. Makarov, and A. K. Motovilov, Perturbation of spectra and spectral subspaces, Trans. Amer. Math. Soc. 359 (2007), no. 1, 77-89, DOI 10.1090/S0002-9947-0603930-4. MR2247883 (2008b:47026)

[8] Konstantin A. Makarov and Albrecht Seelmann, The length metric on the set of orthogonal projections and new estimates in the subspace perturbation problem, J. Reine Angew. Math. 708 (2015), 1-15. MR3420326

[9] A. Seelmann, On an estimate in the subspace perturbation problem, accepted for publication in Journal d'Analyse Mathématique. E-print arXiv:1310.4360 [math.SP] (2013).

[10] Albrecht Seelmann, Notes on the $\sin 2 \Theta$ theorem, Integral Equations Operator Theory 79 (2014), no. 4, 579-597, DOI 10.1007/s00020-014-2127-z. MR.3231246

[11] A. Seelmann, Perturbation theory for spectral subspaces, Dissertation, Johannes GutenbergUniversität Mainz, 2014.

[12] Christiane Tretter, Spectral theory of block operator matrices and applications, Imperial College Press, London, 2008. MR2463978 (2010e:47033)

FB 08 - Institut Für Mathematik, Johannes Gutenberg-Universität Mainz, Staudinger WeG 9, D-55099 MainZ, Germany

E-mail address: seelmann@mathematik.uni-mainz.de 